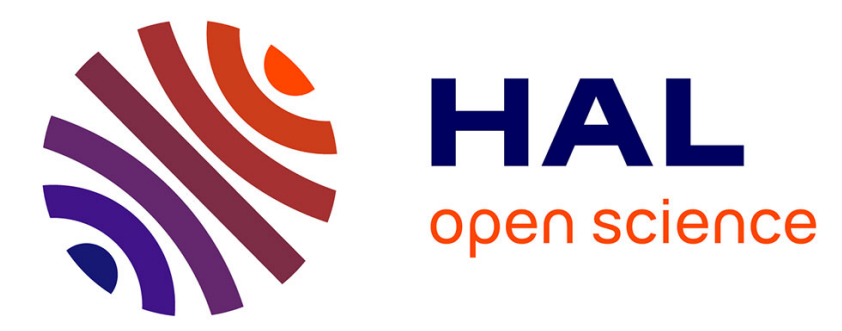

\title{
Foot Function Index: A Promising Questionnaire for Individuals With Charcot-Marie-Tooth Disease Type $1 \mathrm{~A}$
}

Lucie Bihel, Vivien Reynaud, Pascal Givron, Pierre Clavelou, Catherine Cornut-Chauvinc, Bruno Pereira, Eric Thomas, Frederic Taithe, Emmanuel Coudeyre

\section{To cite this version:}

Lucie Bihel, Vivien Reynaud, Pascal Givron, Pierre Clavelou, Catherine Cornut-Chauvinc, et al.. Foot Function Index: A Promising Questionnaire for Individuals With Charcot-Marie-Tooth Disease Type 1A. Archives of Physical Medicine and Rehabilitation, 2019, 100 (12), pp.2403-2406. 10.1016/j.apmr.2019.06.003 . hal-03001619

\section{HAL Id: hal-03001619 https://hal.science/hal-03001619}

Submitted on 21 Dec 2021

HAL is a multi-disciplinary open access archive for the deposit and dissemination of scientific research documents, whether they are published or not. The documents may come from teaching and research institutions in France or abroad, or from public or private research centers.
L'archive ouverte pluridisciplinaire HAL, est destinée au dépôt et à la diffusion de documents scientifiques de niveau recherche, publiés ou non, émanant des établissements d'enseignement et de recherche français ou étrangers, des laboratoires publics ou privés.

\section{(ㅇ)(1) $\$$}

Distributed under a Creative Commons Attribution - NonCommercial - NoDerivatives 44.0 


\section{Foot Function Index in patient with CMT1A}

\section{Foot Function Index: a promising questionnaire for patients with \\ Charcot-Marie-Tooth disease type $1 \mathrm{~A}$.}

Lucie Bihel $^{1}$, MD; Vivien Reynaud ${ }^{1}$, MD-Msc; Pascal Givron ${ }^{1}$, MD; Pierre Clavelou ${ }^{2}$, MD-

$\mathrm{PhD}$; Catherine Cornut-Chauvinc ${ }^{3}, \mathrm{MD}$; Bruno Pereira ${ }^{4}, \mathrm{PhD}$; Eric Thomas ${ }^{5}$; Frederic Taithe ${ }^{2}$, MD; Emmanuel Coudeyre ${ }^{1}, \mathrm{MD}-\mathrm{PhD}$.

(1) Service de Médecine Physique et de Réadaptation, CHU Clermont-Ferrand, INRA, Université Clermont Auvergne, Clermont-Ferrand, France

(2) Service de neurologie, CHU Clermont-Ferrand, INSERM Clermont Auvergne Université, Clermont-Ferrand, France

(3) Clinique Charcot, Sainte-Foy-lès-Lyon, France

(4) Direction Recherche clinique et innovation, CHU de Clermont-Ferrand, France

(5) Boucharenc Podo-orthèse, Saint-Chély d'Apcher, France

Acknowledgments: The authors sincerely thank all the patients who gave their time for this clinical study. They also thank Dr AG Jegu and C Pourtier-Piotte who were involved at the very beginning of the study.

Funding: The authors sincerely thank Clermont-Ferrand University Hospital for funding (2012).

Conflict of Interest: Each individual named as an author meets the uniform Requirements for 
Manuscripts Submitted to biomedical Journals criteria for authorship (ICMJE form). All the authors declare do not have any conflict of interest to declare.

Related author: Pr Emmanuel Coudeyre,

Department of Physical Medicine and Rehabilitation, Clermont-Ferrand University Hospital,

Hôpital Louise Michel, route de Chateaugay,

BP 30056, 63118 Cébazat, France

Tel: + $33473750900 \quad$ Fax: + 33473750901

Email: ecoudeyre@chu-clermontferrand.fr

Clinical Trial: ClinicalTrials.gov Identifier: NCT01750710. 
Comment réduire la mortalité par cancer du poumon chez les personnes vivant avec le VIH ? Du sevrage tabagique au dépistage radiologique

How to reduce lung cancer mortality among people living with HIV?

A-M. Ruppert ${ }^{1-2}$, A. Lavolé ${ }^{1-2}$, A. Makinson ${ }^{3}$, B. Le Maître ${ }^{4}$, J. Cadranel $^{1-2}$

1- $\quad$ GRC n04, Theranoscan, Faculté de Médecine P\&M Curie, Sorbonne Université, Hôpital Tenon (AP-HP), 4 rue de la Chine, F-75252 Paris, France

2- $\quad$ Service de Pneumologie, Unité de Tabacologie, Hôpital Tenon, Assistance PubliqueHôpitaux de Paris

3- Département des Maladies Infectieuses et Unité InsermU1175, Université et CHU de Montpellier, Montpellier

4- $\quad$ Unité de coordination de tabacologie, CHU de Caen

Titre court :

Sevrage tabagique et dépistage radiologique

\section{Auteur-correspondant :}

A.-M. Ruppert,

UF de tabacologie, Service de Pneumologie, Hôpital Tenon, 4 rue de la Chine, 75970, Paris

Cedex 20. Tel : 0156017847 - mail : anne-marie.ruppert@aphp.fr

Reçu le : 17.04 .19

Accepté le : 28.08.19

Déclaration de liens d'intérêts :

Les auteurs déclarent ne pas avoir de liens d'intérêts 


\section{Résumé}

Le cancer bronchique $(\mathrm{CB})$ représente la première cause de décès par cancer chez les personnes vivant avec le VIH (PVVIH). L'exposition au tabac est plus importante chez les PVVIH $(38,5 \%)$ et explique en partie l'augmentation du risque de CB. Pour réduire la mortalité par CB, deux approches sont discutées : le dépistage du CB par scanner thoracique faiblement dosé et le sevrage tabagique.

Le dépistage par scanner thoracique est réalisable chez les PVVIH. Le taux de faux positifs n'est pas plus important qu'en population générale, sauf en cas de CD4 <200/mm³ L'impact sur la survie reste à évaluer.

Malgré la forte prévalence de fumeur, la recherche sur le sevrage tabagique chez les PVVIH est rare. Des données de très faible qualité, issues de 11 études, ont montré que des interventions plus intensives pour arrêter de fumer permettaient de parvenir à une abstinence à court terme. Un seul essai randomisé de phase 3 a montré la supériorité de la varénicline contre placebo sur le sevrage tabagique à long terme.

Le bénéfice maximum sur la réduction de la mortalité spécifique par CB pourrait être observé par le sevrage tabagique et le dépistage par scanner thoracique.

Mots clés : $4 \mathrm{CB}$, tabac, VIH, dépistage 


\section{How to reduce lung cancer mortality among people living with HIV?}

\section{Abastract}

Lung cancer is the leading cause of cancer related death among people living with HIV (PLHIV). Tobacco exposure is higher among PLHIV (38.5\%) and mainly explains the increased risk of lung cancer. To reduce lung cancer mortality, two approaches need to be implemented: lung cancer screening with low-dose thoracic CT scan and smoking cessation.

Low dose CT scan is feasible in PLHIV. The false positive rate is not higher than in the general population, except for cases with CD4 $<200 / \mathrm{mm} 3$. The impact on survival remains to be assessed.

Despite the high prevalence, smoking cessation research among PLHIV is scarce. Very low quality data from 11 studies showed that more intensive smoking cessation interventions were effective in achieving short-term abstinence. A single randomized phase 3 trial showed the superiority of varenicline compared to placebo in long-term smoking cessation.

The maximum benefit of reducing lung cancer mortality should be obtained by combining smoking cessation and lung cancer screening.

Key Words: Lung cancer, tobacco, HIV, screening 


\section{Introduction}

En France, près de 150000 personnes vivent avec le VIH (PVVIH) et 6600 nouveaux cas ont été détectés en 2014 [1]. L'incidence et la mortalité par cancer chez les PVVIH ont évolué depuis les années 1990. Les complications associées au VIH ne représentent plus qu'un quart des décès dans cette population [2][3]. Cette évolution est liée à l'émergence de combinaisons antirétrovirales très actives, ayant permis d'allonger l'espérance de vie des patients et de diminuer les complications liées au VIH. Ainsi les cancers sont devenus la première cause de mortalité devant les maladies cardio-vasculaires.

Les objectifs de cet article sont de discuter les données spécifiques concernant le dépistage du cancer bronchique (CB) et du sevrage tabagique chez les PVVIH.

\section{Les caractéristiques du CB chez le PVVIH}

Le cancer bronchique est le plus fréquent des cancers non classant SIDA et représente la première cause de décès par cancer chez les PVVIH. Le traitement du CB chez les PVVIH peut sembler plus complexe que dans la population générale, en raison d'interactions médicamenteuses avec les antirétroviraux, d'une majoration possible de l'agressivité tumorale et de comorbidités multiples [4]. Les facteurs de risque de CB incriminés chez les PVVIH sont comparables à ceux de la population générale. L'exposition au tabac est plus importante chez les PVVIH que dans la population générale [5] et explique en très grande partie l'augmentation du risque de CB chez les PVVIH par rapport à la population générale.

Certaines études ont montré une augmentation du risque de CB chez les PVVIH indépendamment du tabac. Des hypothèses sont avancées: le rôle de l'inflammation, chronique suite aux infections à répétition, la prévalence plus élevée de BPCO chez les PVVIH, qui est un facteur de risque indépendant de CB en population générale [6] et surtout l'immunodépression et son corrélat potentiel d'inflammation in situ majorée par rapport à la population générale après exposition au tabac [7]. Une méta-analyse, réalisée sur des patients 
infectés par le VIH (7 études) et des patients transplantés (5 études), montrait une augmentation du risque de cancer dans ces populations [8]. Ce risque augmenterait avec la diminution des CD4, de même que la diminution du ratio CD4/CD8.

Si le risque de CB est plus élevé chez les PVVIH, cet excès de risque semble toutefois diminuer avec le temps depuis l'avènement des combinaisons antirétrovirales. Une étude nord-américaine de registres de cancers et d'infections par le VIH a montré un déclin significatif de 2,8\% du taux standardisé de l'incidence du CB entre 1996 et 2010, associé à un déclin significatif entre les mêmes périodes de l'estimation du risque de 4,4\% [9]. L'étude de 84504 PVVIH de la base de données de la fédération hospitalière française (FHDH) confirme ces données avec une diminution du risque de CB dans la période 1997-2000 de 4,7, dans la période 2000-2004 de 3,8, et dans la période 2005-2009 de 2,8 [10].

Les explications possibles de cette réduction du risque du CB chez les PVVIH sont multiples : - un taux médian de CD4 en augmentation chez les PVVIH grâce aux recommandations de traitement antirétroviral de plus en plus précoce, et donc une amélioration sous-jacente de l'immunité antitumorale

- une diminution, comme en population générale, de la prévalence du tabagisme actif. La prévalence du tabagisme actif entre les différentes études transversales en France semblent en diminution au cours des années de 40-50\% 2005 à 37,5\% en 2011 [11][12].

- et une réduction de l'incidence des pneumopathies récurrentes chez les PVVIH: les infections sont responsable d'une inflammation chronique et favorisent le développement du CB $[13,14]$

L'immunité est contrôlée au moment du diagnostic de CB et la plupart des patients sont sous trithérapie antirétrovirale. La répartition histologique est similaire à celle de la population générale avec une prépondérance d'adénocarcinome, suivi de cancer épidermoïde. Dans certaines études le pronostic est moins bon que celui de la population générale après ajustement aux traitement et au stade $[13,15]$. Cependant, ces résultats peuvent aussi refléter 
des systèmes de prise en charge sociale différents avec parfois des difficultés d'accès aux traitements. Une étude a montré que le pronostic du CB chez les PVVIH était identique par rapport à la population générale [10].

La stratégie de prise en charge du CB chez le patient VIH est identique à celle de la population générale, même s'ils sont classiquement exclus des essais thérapeutiques. Néanmoins, les interactions médicamenteuses des antirétroviraux doivent être prises en compte et peuvent diminuer l'efficacité de la chimiothérapie ou augmenter sa toxicité. Des recommandations d'expert sur la prise en charge du CB chez les PVVIH ont été récemment publiées [16]. Afin d'optimiser la prise en charge des PVVIH atteints de cancer, une Réunion de Concertation Pluridisciplinaire nationale ONCOVIH, composée d'infectiologue, de pharmacologue, d'oncologue, de pneumologues, est proposée deux fois par mois (https://www.cancervih.org/).

-Le cancer bronchique est le plus fréquent des cancers non classant SIDA et représente la première cause de décès par cancer chez les patients vivant avec le VIH (PVVIH).

-Le traitement du CB chez les PVVIH est plus complexe que dans la population générale, en raison de comorbidités multiples, d'interactions médicamenteuses avec les antirétroviraux, d'une majoration possible de l'agressivité tumorale.

-En dehors du tabagisme, le risque augmenté de $\mathrm{CB}$ chez ces patients serait lié à l'inflammation chronique, à la présence d'une BPCO associée, à l'immunodépression; ce risque semble diminuer depuis l'utilisation d'antirétroviraux.

La stratégie de prise en charge du CB chez le patient VIH est identique à celle de la population générale; en raison des spécificités liées aux interactions médicamenteuses, une Réunion de Concertation Pluridisciplinaire nationale ONCOVIH, est proposée deux fois par mois (https://www.cancervih.org/).

\section{Dépistage du CB}

L'objectif du dépistage du CB est de découvrir la maladie à un stade précoce pour opérer plus de patients et améliorer le pronostic. L'étude du National Lung Cancer Screening Trial (NLST) a montré dans la population générale (âge 55 à 74 ans, fumeurs de plus de 30 paquets/années) que le dépistage du CB par scanner thoracique faiblement dosé permettait de réduire la mortalité spécifique par CB de $20 \%$ et mortalité globale de $7 \%$ dans la population générale [17]. Cette année, ont été rapporté les résultats de l'étude NELSON qui confirme une diminution de la mortalité spécifique par le scanner thoracique de $26 \%$ chez les hommes (IC 
95\%:0,6-0,91: $\mathrm{p}=0,003$ ) et davantage chez les femmes (IC 95\%:0,35-1,04: $\mathrm{p}=0,05$ ) de plus de 50 ans avec un tabagisme de plus de 25 ans [18]. Le protocole de l'étude NELSON se basant sur volume et le temps de doublement de volume permettait de réduire le nombre de faux-positifs $(2,2 \%$ de tests positifs dans NELSON versus $25 \%$ dans NLST, dont pour NELSON $41 \%$ de faux-positifs et NLST $96 \%$ de faux-positifs). Suite à ces données européennes et la réduction des faux-positifs, la position de la Haute Autorité de Santé (HAS) sur le dépistage, actuellement non recommandé en France, devrait changer [19].

\section{Dépistage du CB chez la personne vivant avec le VIH}

Parmi les 14 citations fournies sur pubmed par l'équation de recherche «VIH et dépistage du $\mathrm{CB} »$, trois articles sur le dépistage ont été retenus (Tableau 1).

Le premier essai de dépistage du CB par scanner thoracique annuel a été réalisé aux Etats Unis entre 2006 à 2013 chez 224 fumeurs ou fumeurs sevrés infectés par le VIH [20]. Cette étude souligne l'importance du choix des critères d'éligibilité pour l'efficacité d'une stratégie de dépistage du CB par scanner faiblement dosé [20]. Les critères d'inclusion étaient l'absence de symptômes évocateurs de CB, un âge $\geq 25$ ans, un tabagisme $\geq 20$ paquet-années actif ou sevré depuis moins de 15 ans. Cette étude a inclus 224 sujets d'âge médian de 48 ans (44-53 ans), majoritairement des hommes (72\%), d'origine Afro-américaine pour $90 \%$ et fumeurs actifs pour 39,7\% (médiane de 34 paquet-années). De plus, $40 \%$ des participants avaient une histoire de consommation de marijuana, 29\% de cocaïne, et 58\% un antécédent d'usages de drogues intraveineuses. La médiane du nadir de CD4 était de 179 cellules/ $\mu 1$, la médiane du dernier taux de CD4 de 400 cellules/ $\mu 1$, et $59 \%$ avaient une charge virale $<400$ copies/ml. Un scanner thoracique faiblement dosé annuel a été réalisé pendant 5 ans. Dans cette étude, un nodule était considéré comme positif si son diamètre était $\geq 4 \mathrm{~mm} ; 14 \%$ des sujets inclus avaient un nodule significatif [20]. Aucun CB ne fut dépisté, probablement en 
raison du faible nombre de sujets inclus, mais surtout de l'âge très jeune d'une grande partie des sujets, et de la mauvaise adhésion au protocole de dépistage.

L'étude ANRS EP48 «HIV chest» a évalué en France la faisabilité du dépistage du CB parmi les PVVIH [21]. Au total, 442 patients étaient inclus avec un âge médian de 49,8 ans et une consommation de tabac de 30 paquets-années. Parmi ces patients, $21 \%(\mathrm{n}=94)$ présentaient une anomalie radiologique nécessitant dans 3,4\% des cas une procédure diagnostique invasive. Un CB était diagnostiqué chez 2,3\% des patients $(n=10)$, dont $2 \%$ (n=9) par scanner. Un seul cas de CB est diagnostiqué au cours de l'étude avec un scanner initial de dépistage normal (cas de cancer bronchique à petites cellules disséminés). Le dépistage par scanner est faisable. Néanmoins, l'impact sur la survie chez les PVVIH reste à évaluer.

Enfin, une étude de cohorte danoise, réalisée chez 901 PVVIH, dont 113 à haut risque de $\mathrm{CB}$ (> 50 ans, > $30 \mathrm{PA})$, a retrouvé un taux élevé de $\mathrm{CB}$ uniquement chez les personnes à haut risque [22]. Parmi les personnes à haut risque, $11(9,7 \%)$ présentaient une anomalie radiologique, 9 (tous dans le groupe à haut risque) bénéficiaient d'une procédure diagnostique invasive ce qui a permis d'identifier un taux de 2,7 \% (3/113) de cancer bronchopulmonaire dans le groupe à haut risque.

Les résultats concernant la prévalence de nodules sur les TDM chez les PVVIH semblent rassurants, puisque la prévalence des nodules significatifs était dans l'intervalle de celle retrouvée dans les études en population générale [20,21].

Cependant dans l'étude NLST, l'arrêt du tabac dans le bras contrôle permettait dès la $7^{\text {ième }}$ année de réduire la mortalité spécifique par $\mathrm{CB}$ au même niveau que le dépistage du $\mathrm{CB}$ [23]. Le bénéfice maximum serait observé avec une combinaison de sevrage tabagique et de dépistage par TDM thoracique faible dose, avec une réduction estimée de $38 \%$ de la mortalité spécifique par CB dans l'étude NLST [23].

-L'étude du National Lung Cancer Screening Trial (NLST) et l'étude NELSON ont montré dans la population générale (âge 55 à 74 ans, fumeurs de plus de 30 paquets/années) que le 
dépistage du $\mathrm{CB}$ par scanner thoracique faiblement dosé permettait de réduire la mortalité spécifique par CB et la mortalité globale dans la population générale.

- La position de la Haute Autorité de Santé (HAS) sur le dépistage, actuellement non recommandé en France, devrait changer.

-Plusieurs études spécifiques de dépistage du CB, chez les PVVIH ont été réalisées. Le dépistage par scanner thoracique est réalisable chez les PVVIH. Le taux de faux positifs n'est pas plus important qu'en population générale, sauf en cas de CD4 <200/mm3. L'impact sur la survie reste à évaluer.

\section{Tabac et VIH}

La prévalence du tabagisme a diminué en France avec actuellement 26,9\% de fumeurs quotidien [24]. Néanmoins, la proportion de fumeurs parmi les PVVIH reste élevée. L'étude VESPA-2 (2011) analysait les conditions de vie des PVVIH suivies en France et montrait un tabagisme actif de 37,5\% [11], à mettre en perspective avec des taux de tabagisme active entre 40 et 50\% quelques années plus tôt [12]. D'après une analyse systématique réalisée à partir de 51 études, la prévalence du tabagisme chez les PVVIH est de 36\% chez les femmes et 50\% chez les hommes [25]. La prévalence du tabagisme est plus importante chez les PVVIH par rapport à la population générale.

Le tabagisme varie fortement entre les différents groupes socio-épidémiologiques. Ainsi, dans l'enquête ANRS-EN20-mortalité 2010, le tabagisme actif ou sevré était de 96,6\% dans le groupe usagers de drogues intraveineuses, 74,7\% dans le groupe hommes qui ont des rapports sexuels avec d'autres hommes, 63,6\% dans le groupe hétérosexuel français et de 31,1\% dans le groupe hétérosexuel étranger [3].

L'impact du tabac sur la survie a été évalué chez 17995 PVVIH [26]. Dans cette cohorte, $60 \%$ des personnes étaient fumeuses ou ex-fumeuses. Le rapport de taux de mortalité des fumeurs ou ex-fumeurs par rapport aux non-fumeurs était de 1,94 [95\% IC : 1,56-2,41]. Cet excès de mortalité était liée aux maladies cardio-vasculaires [6,28 (95\% IC 2,19-18,0)] et cancers non classant SIDA [3,31 (95\% IC 1,80-5,45)]. Ainsi chez un homme fumeur de 35 ans vivant avec le VIH, l'impact du tabac sur la diminution de la survie était estimé à 7,9 ans 
(95\% CI 7,1-8,7), alors que l'impact du VIH sur la survie était de 5,9 ans (95\% CI 4,9-6,9) [26].

Il y a peu d'études sur l'arrêt du tabac chez les PVVIH. Cependant, la stratégie du sevrage chez le fumeur est peu modifiée par la présence d'un diagnostic du VIH. Les deux modalités qui ont prouvé leur efficacité sont le traitement pharmacologique, par substituts nicotiniques, varénicline ou bupropion, ainsi que le suivi en consultation de tabacologie.

De nombreuses études et méta-analyses ont prouvé l'efficacité des substituts nicotiniques versus placebo qui permettent de multiplier par deux le pourcentage de succès de sevrage à 1 an (20\% versus 10\%) [27]. La varénicline, un agoniste partiel des récepteurs nicotiniques $\alpha 4 \beta 2$, augmente les chances de succès de sevrage tabagique à long terme [28]. La varénicline est remboursée en $2^{\text {ième }}$ intention après échec des substituts nicotiniques chez les fumeurs fortement dépendant (test de Fagerström $\geq 7$ )[28]. Il n'existe pas d'interaction médicamenteuse entre la varénicline et les antirétroviraux, en particulier par le CYP 450. Le bupropion est un inhibiteur de la capture de la dopamine et de la noradrénaline. Il est peu utilisé dans le sevrage tabagique en raison des nombreuses contre-indications : antécédents de convulsion, sevrage alcoolique et interaction avec les médicaments métabolisés par le cytochrome P450, dont les antirétroviraux. En raison de l'absence de données d'efficacité supérieure aux autres traitements et de tolérance chez les PVVIH, il n'est pas recommandé de prescrire cette molécule.

L'objectif des thérapies cognitives et comportementales réalisées au cours des consultations de tabacologie est d'aider le fumeur à se débarrasser de son comportement de fumeur par apprentissage d'un nouveau comportement. Cette prise en charge fait partie des recommandations de la Haute Autorité de Santé (HAS) [29]. L'acupuncture, l'hypnose, l'homéopathie sont utilisés depuis longue date dans le sevrage tabagique. Ces techniques ne sont pas reconnues comme efficaces. 
-La prévalence du tabagisme est plus importante chez les PVVIH par rapport à la population générale : 37,5\% de fumeurs actifs (dans l'étude VESPA-2).

-L'impact du tabac sur la survie du PVVIH a été évalué : la mortalité des fumeurs ou exfumeurs par rapport aux non-fumeurs est doublée.

-La stratégie de sevrage tabagique chez ce fumeur PVVIH, est peu modifiée par la présence d'un diagnostic du VIH : substituts nicotiniques, varénicline (pas d'interactions médicamenteuses avec les antirétroviraux, bonne tolérance) ou bupropion (présence d'interactions médicamenteuses), thérapies cognitives et comportementales, suivi en consultation de tabacologie).

\section{Sevrage tabagique chez la personne vivant avec le VIH}

Parmi les citations fournies par l'équation de recherche «VIH et sevrage tabagique » sur pubmed (>800), 12 études inclus dans la méta-analyse Cochrane publiée en 2016 [30] ont été retenues. Depuis 2016, deux études françaises ont été publiées sur ce sujet et sont discutées.

Malgré la forte prévalence des fumeurs, la recherche sur le sevrage tabagique chez les PVVIH est étonnamment rare. Il n'existe à ce jour que peu de données de haute qualité sur les interventions pour lutter contre le tabagisme chez les PVVIH.

La méta-analyse Cochrane comporte 12 études pertinentes avec 2087 sujets [30]. Toutes les études prévoyaient une intervention associant un soutien comportemental et une pharmacothérapie, comparée dans la plupart des études à un contrôle moins intensif, comprenant généralement une brève intervention comportementale et un traitement pharmacologique. L'ensemble des études, à l'exception d'une étude suisse, étaient réalisée aux Etats-Unis. L'abstinence à long terme regroupée était de $8 \%$ dans les conditions d'intervention et de contrôle [30] (tableau 2). Des données de très faible qualité, issues de 11 études, ont montré que des interventions plus intensives pour arrêter de fumer permettaient de parvenir efficacement à une abstinence à court terme [risque relatif $1,51,95 \%$ IC (1,15 à 2,00)]. L'abstinence à court terme était de 13\% (118/937) dans le groupe d'intervention versus 8\% (67/848) dans le groupe témoin. L'analyse en sous-groupes ne permettait pas d'identifier un traitement pharmacologique présentant une efficacité supérieure, en comparant substituts nicotiniques versus varénicline. 
En 2016, ont été rapporté les résultats du seul essai randomisé de phase 3 en double aveugle comparant un traitement par varénicline associé à des conseils de sevrage tabagique pendant 3 mois contre placebo associé à des conseils de sevrage tabagique [31]. A la fin de l'étude à 48 semaines, significativement plus de participants était abstinents dans le groupe varénicline [18/123, soit 15\%, IC 95\% (8-21)] par rapport au groupe placebo [8/124, soit 6\%, IC 95\% (2-11) ( $\mathrm{p}=0,036)$. Il s'agit d'une première étude montrant un bénéfice à long terme d'une intervention de sevrage tabagique chez les PVVIH. Par ailleurs, la tolérance sous varénicline était excellente, sans augmentation de l'incidence des événements psychiatriques ou cardiovasculaires. Les effets indésirables les plus fréquents étaient les nausées, l'insomnie et les cauchemars.

Récemment ont été rapporté les résultats d'une cohorte de PVVIH au CHU de Caen comprenant une prise en charge du tabac en lien étroit entre le service de maladies infectieuses et de tabacologie [32]. Dans cette cohorte, 280 patients (39\%) vivant avec le VIH étaient fumeurs. La moitié (147, soit 53\%) acceptait de participer au programme de sevrage tabagique. Au cours du suivi, 41 fumeurs (28\%) se sont retirés du programme. Après 6 mois, $60(57 \%)$ des 106 fumeurs qui ont terminé l'intervention avaient cessé de fumer et étaient plus susceptible d'utiliser la varénicline [32].

Il n'existe pas de données concernant la cigarette électronique dans le sevrage tabagique chez les PVVIH. La démarche de réduction de risque par l'utilisation d'une cigarette électronique semble particulièrement intéressante dans cette population et cette approche devra être évaluée au cours d'études prospectives [33]. Néanmoins, à ce jour, les professionnels de santé restent prudents avant de recommander la cigarette électronique chez les PVVIH [34].

-Peu de données de haute qualité existent sur les interventions pour lutter contre le tabagisme chez les PVVIH.

-Il faut toujours associer un soutien comportemental et une pharmacothérapie; les taux d'abstinence tabagique restent peu élevés à long terme (sauf études spécifiques avec forte interaction infectiologue-tabacologue)

-Pas d'études sur la cigarette électronique dans le sevrage tabagique chez les PVVIH. 


\section{Discussion}

Pour réduire la mortalité par $\mathrm{CB}$, deux approches complémentaires doivent être mises en place ; d'une part le dépistage du CB par scanner thoracique faiblement dosé et d'autre part le sevrage tabagique.

Le dépistage par scanner thoracique faiblement dosé est réalisable chez les PVVIH. Dans l'étude française, le taux de faux positifs n'était pas plus important que dans l'étude en population générale européenne NELSON [19]. Cependant davantage de nodules sont découverts en cas de $\mathrm{CD} 4<200 / \mathrm{mm}^{3}$ [35]. Ainsi la crainte de nodules nécessitant des procédures diagnostiques invasives et révélant des comorbidités infectieuses ne semble pas fondée chez les PVVIH avec un taux de CD4>200/ $\mathrm{mm}^{3}$.

Néanmoins, l'impact sur la survie chez les PVVIH reste à évaluer. La communication récente des résultats positifs de l'étude européenne NELSON, réalisée dans la population générale de fumeurs de plus de 50 ans et plus de 25 paquets-année, devrait modifier la position de la HAS sur le dépistage, actuellement non recommandé en France [19], mais fortement plébiscité par l'Intergroupe Francophone de Cancérologie Thoracique (IFCT) [36]. S'il faut abaisser l'âge du début du dépistage du CB chez les PVVIH par rapport aux recommandations de l'étude NELSON, soit avant 50 ans, reste à définir. En effet, l'âge au diagnostic de CB chez les PVVIH par rapport à la population générale est de 4 ans plus jeune environ $[37,38]$.

La participation à un essai de dépistage du CB peut favoriser l'arrêt du tabac. Cependant, certains patients rapportent une poursuite du tabagisme avec un «permis » de fumer en cas de normalité du scanner thoracique [39]. Par ailleurs, l'arrêt du tabac permet de réduire la mortalité spécifique par $\mathrm{CB}$ au même niveau que le dépistage du $\mathrm{CB}$ dès la $7^{\text {ième }}$ année (20). Le bénéfice maximum pourrait être observé avec une combinaison de sevrage tabagique et de dépistage par TDM thoracique faible dose, avec une réduction estimée de 38\% de la mortalité spécifique par CB (20). La stratégie optimale de sevrage tabagique chez les 
patients participant à un dépistage par scanner thoracique demeure incertaine. Les futures études devraient être axées sur l'évaluation de l'efficacité et de la mise en œuvre de conseils combinés à une pharmacothérapie afin d'optimiser le sevrage tabagique pendant le dépistage du CB [40].

Malgré la forte prévalence du tabagisme, peu de données de haute qualité concernant le sevrage tabagique chez les PVVIH sont disponibles. Mercié et col, ont montré en France au cours d'un essai de phase III randomisé en double aveugle la supériorité de la varénicline au placebo dans cette population [41]. La varénicline est sans danger pour les fumeurs vivant avec le VIH présentant une comorbidité psychiatrique, y compris la dépression sévère ou des complications cardio-vasculaires [28]. Dans cette étude, la prescription de la varénicline par l'infectiologue aboutissait à presque deux fois plus de patients sevrés en tabac comparée à la prescription réalisée par les tabacologues. Cette meilleure efficacité pourrait être liée au lien particulier de confiance des PVVIH envers leur médecin infectiologue référent et sa capacité du médecin à livrer un message personnalisé. Une collaboration étroite entre spécialistes et tabacologues est nécessaire dans la prise en charge de la personne fumeuse vivant avec le VIH en raison de la dépendance importante et des co-addictions ou comorbidités psychiatriques fréquentes.

En France actuellement, près de $40 \%$ des PVVIH sont fumeurs actifs avec une dépendance élevée au test de Fagerström [42]. Dans une étude à Montpellier, une consommation excessive d'alcool était notée chez $22 \%$ des PVVIH, une consommation de cannabis chez $27,7 \%$, mais également une consommation de poppers $(16,4 \%)$, de cocaïne $(8,9 \%)$, de psychotropes $(7,1 \%)$, ou de GHB/GBL (4,7\%)[43]. Ces co-addictions sont des facteurs d'échec au sevrage tabagique reconnues, tout comme les comorbidités psychiatriques. La prévalence de la dépression était de 28,1\% chez les PVVIH, allant de 10,9 à 55,7\% selon les différents groupes étudiés [44]. 
La place de la cigarette électronique dans le sevrage tabagique chez la PVVIH reste à définir. Il n'existe pas de données dans cette population. Dans l'analyse portant sur 3 essais randomisés contrôlés incluant 1246 fumeurs en dehors de tout contexte d'infection avec VIH, la cigarette électronique multiplie par 2 le taux d'arrêt du tabac [45]. Néanmoins, la qualité des preuves est incertaine en raison du faible nombre de fumeurs inclus et du plan d'analyse des différentes études. Récemment était publié un essai sur 886 fumeurs montrant un taux de sevrage tabagique à 1 an à $18 \%$ avec la cigarette électronique versus 9,9\% avec les substituts nicotiniques [46]. L'HAS, suite au rapport sur la cigarette électronique du Public Health England, considère la cigarette électronique comme un outil de réduction des risques pour les populations fumeuses désireuses d'arrêter leur consommation de tabac.

Chez les personnes infectées par le VIH, un dépistage systématique du tabac doit être réalisé et une prise en charge proposée. Cette prise en charge doit être effectuée en lien étroit entre tabacologue et médecin référent. Il doit comporter un traitement pharmacologique. Ainsi dans le programme de Caen, la moitié des fumeurs vivant avec le VIH et qui participaient au programme étaient sevré à 6 mois [32].

-Pour réduire la mortalité par $\mathrm{CB}$, deux approches complémentaires doivent être mises en place : le dépistage du $\mathrm{CB}$ par scanner thoracique faiblement dosé et le sevrage tabagique.

-Pour le dépistage du CB chez les PVVIH, l'âge recommandé devrait être abaissé par rapport aux critères des études NLST ou Nelson.

-Pour les futures études, il faudra évaluer l'efficacité de conseils combinés à une pharmacothérapie pour optimiser le sevrage tabagique au cours du dépistage du CB.

-Nécessité d'une collaboration étroite entre infectiologues, tabacologues et pneumologues.

\section{Points forts de l'article :}

-Le cancer bronchique est la première cause de décès par cancer chez les personnes vivant avec le VIH.

-Ces patients fument très souvent, expliquant en partie l'augmentation du risque de CB.

-Pour réduire la mortalité par $\mathrm{CB}$, deux approches sont discutées : le dépistage du CB par scanner thoracique faiblement dosé et le sevrage tabagique.

a) Le dépistage par scanner thoracique est possible avec des taux de faux positifs pas plus important qu'en population générale, sauf en cas de CD4 très abaissés.

b) Le sevrage tabagique chez ces patients paraît rare. Pourtant des interventions intensives pour arrêter de fumer permettaient de parvenir à une abstinence à court terme. Pour le sevrage 
tabagique à long terme, la supériorité de la varénicline contre placebo est retrouvée dans un seul essai.

-Le bénéfice maximum sur la réduction de la mortalité spécifique par $\mathrm{CB}$ pourrait être observé par le sevrage tabagique et le dépistage par scanner thoracique. 


\section{Références}

[1] Prévalence du VIH / Infection à VIH et sida / VIH-sida / IST / Maladies infectieuses / Dossiers thématiques / Accueil n.d. http://invs.santepubliquefrance.fr/Dossiersthematiques/Maladies-infectieuses/VIH-sida-IST/Infection-a-VIH-et-sida/Prevalencedu-VIH (accessed November 14, 2018).

[2] Morlat P, Roussillon C, Henard S, Salmon D, Bonnet F, Cacoub P, et al. Causes of death among HIV-infected patients in France in 2010 (national survey): trends since 2000. AIDS 2014;28:1181-91.

[3] Lert F. Caractéristiques sociales et comportementales des personnes séropositives pour le VIH décédées en 2010 en France métropolitaine : quelles implications pour la prise en charge ? 2016:6.

[4] Domblides C, Canellas A, Wislez M, Fallet V, Antoine M, Crequit P, et al. [Lung cancer in HIV-infected patients]. Bull Cancer 2018;105:111-9.

[5] Mdodo R, Frazier EL, Dube SR, Mattson CL, Sutton MY, Brooks JT, et al. Cigarette smoking prevalence among adults with HIV compared with the general adult population in the United States: cross-sectional surveys. Ann Intern Med 2015;162:335-44.

[6] Makinson A, Hayot M, Eymard-Duvernay S, Ribet C, Raffi F, Pialoux G, et al. HIV is associated with airway obstruction: a matched controlled study. AIDS 2018;32:227-32.

[7] Guiguet M, Boué F, Cadranel J, Lang J-M, Rosenthal E, Costagliola D, et al. Effect of immunodeficiency, HIV viral load, and antiretroviral therapy on the risk of individual malignancies (FHDH-ANRS CO4): a prospective cohort study. Lancet Oncol 2009;10:1152-9.

[8] Grulich AE, van Leeuwen MT, Falster MO, Vajdic CM. Incidence of cancers in people with HIV/AIDS compared with immunosuppressed transplant recipients: a metaanalysis. Lancet 2007;370:59-67.

[9] Robbins HA, Shiels MS, Pfeiffer RM, Engels EA. Epidemiologic contributions to recent cancer trends among HIV-infected people in the United States. AIDS 2014;28:881-90. doi:10.1097/QAD.0000000000000163.

[10] Hleyhel M, Hleyhel M, Bouvier AM, Belot A, Tattevin P, Pacanowski J, et al. Risk of non-AIDS-defining cancers among HIV-1-infected individuals in France between 1997 and 2009: results from a French cohort. AIDS 2014;28:2109-18. doi:10.1097/QAD.0000000000000382.

[11] Tron L, Lert F, Spire B, Dray-Spira R, ANRS-Vespa2 study group. Tobacco smoking in HIV-infected versus general population in france: heterogeneity across the various groups of people living with HIV. PLoS ONE 2014;9:e107451.

[12] Duval X, Baron G, Garelik D, Villes V, Dupré T, Leport C, et al. Living with HIV, antiretroviral treatment experience and tobacco smoking: results from a multisite crosssectional study. Antivir Ther (Lond) 2008;13:389-97.

[13] Hessol NA, Martínez-Maza O, Levine AM, Morris A, Margolick JB, Cohen MH, et al. Lung cancer incidence and survival among HIV-infected and uninfected women and men. AIDS 2015;29:1183-93. doi:10.1097/QAD.0000000000000690.

[14] Shebl FM, Engels EA, Goedert JJ, Chaturvedi AK. Pulmonary infections and risk of lung cancer among persons with AIDS. J Acquir Immune Defic Syndr 2010;55:375-9.

[15] D'Jaen GA, Pantanowitz L, Bower M, Buskin S, Neil N, Greco EM, et al. Human immunodeficiency virus-associated primary lung cancer in the era of highly active antiretroviral therapy: a multi-institutional collaboration. Clin Lung Cancer 2010;11:396-404.

[16] Spano J-P, Poizot-Martin I, Costagliola D, Boué F, Rosmorduc O, Lavolé A, et al. NonAIDS-related malignancies: expert consensus review and practical applications from the multidisciplinary CANCERVIH Working Group. Ann Oncol 2016;27:397-408. 
[17] Results of initial low-dose computed tomographic screening for lung cancer. - PubMed NCBI n.d. https://www-ncbi-nlm-nih-gov.gate2.inist.fr/pubmed/23697514 (accessed November 26, 2018).

[18] Bunn B, Bunn B. NELSON Study Shows CT Screening for Nodule Volume Management Reduces Lung Cancer Mortality by 26 Percent in Men n.d.:2.

[19] Rapport HAS. Pertinence du dépistage du cancer broncho-pulmonaire en France. Point de situation sur les données disponibles et analyse critique des études contrôlées randomisées. Https://WwwHas-SanteFr/Portail/Jcms/C_2001613/Fr/Pertinence-DuDepistage-Du-Cancer-Broncho-Pulmonaire-En-France-Point-de-Situation-Sur-LesDonnees-Disponibles-Analyse-Critique-Des-Etudes-Controlees-Randomisees 2016.

[20] Hulbert A, Hooker CM, Keruly JC, Brown T, Horton K, Fishman E, et al. Prospective $\mathrm{CT}$ screening for lung cancer in a high-risk population: HIV-positive smokers. J Thorac Oncol 2014;9:752-9.

[21] Makinson A, Eymard-Duvernay S, Raffi F, Abgrall S, Bommart S, Zucman D, et al. Feasibility and efficacy of early lung cancer diagnosis with chest computed tomography in HIV-infected smokers. AIDS 2016;30:573-82.

[22] Ronit A, Kristensen T, Klitbo DM, Gelpi M, Kalhauge A, Benfield T, et al. Incidental lung cancers and positive computed tomography images in people living with HIV. AIDS 2017;31:1973-7.

[23] Tanner NT, Kanodra NM, Gebregziabher M, Payne E, Halbert CH, Warren GW, et al. The Association between Smoking Abstinence and Mortality in the National Lung Screening Trial. Am J Respir Crit Care Med 2015;193:534-41.

[24] Pasquereau A. La consommation de tabac en france: premiers résultats du baromètre santé 2017 / tobacco consumption in france: preliminary results from the 2017 health barometeR n.d.:9.

[25] Weinberger AH, Smith PH, Funk AP, Rabin S, Shuter J. Sex Differences in Tobacco Use Among Persons Living With HIV/AIDS: A Systematic Review and Meta-Analysis. J Acquir Immune Defic Syndr 2017;74:439-53.

[26] Helleberg M, May MT, Ingle SM, Dabis F, Reiss P, Fätkenheuer G, et al. Smoking and life expectancy among HIV-infected individuals on antiretroviral therapy in Europe and North America. AIDS 2015;29:221-9.

[27] Stead LF, Perera R, Bullen C, Mant D, Hartmann-Boyce J, Cahill K, et al. Nicotine replacement therapy for smoking cessation. Cochrane Database Syst Rev 2012;11:CD000146..

[28] Anthenelli RM, Benowitz NL, West R, St Aubin L, McRae T, Lawrence D, et al. Neuropsychiatric safety and efficacy of varenicline, bupropion, and nicotine patch in smokers with and without psychiatric disorders (EAGLES): a double-blind, randomised, placebo-controlled clinical trial. Lancet 2016;387:2507-20.

[29] Dépistage du tabagisme et prévention des maladies liées au tabac. Haute Autorité de Santé n.d. https://www.has-sante.fr/jcms/c_2635050/fr/depistage-du-tabagisme-etprevention-des-maladies-liees-au-tabac (accessed August 9, 2019).

[30] Interventions for tobacco use cessation in people living with HIV and AIDS. - PubMed NCBI n.d. https://www-ncbi-nlm-nih-gov.gate2.inist.fr/pubmed/27292836 (accessed October 17, 2018).

[31] Mercié P, Arsandaux J, Katlama C, Ferret S, Beuscart A, Spadone C, et al. Efficacy and safety of varenicline for smoking cessation in people living with HIV in France (ANRS 144 Inter-ACTIV): a randomised controlled phase 3 clinical trial. Lancet HIV 2018;5:e126-35.

[32] Parienti J-J, Merzougui Z, de la Blanchardière A, Dargère S, Feret $P$, Le Maitre $B$, et al. A Pilot Study of Tobacco Screening and Referral for Smoking Cessation Program among HIV-Infected Patients in France. J Int Assoc Provid AIDS Care 2017;16:467-74. 
[33] Bell S, Dean J, Gilks C, Boyd MA, Fitzgerald L, Mutch A, et al. Tobacco Harm Reduction with Vaporised Nicotine (THRiVe): The Study Protocol of an Uncontrolled Feasibility Study of Novel Nicotine Replacement Products among People Living with HIV Who Smoke. Int J Environ Res Public Health 2017;14.

[34] Bell SK, Mena G, Dean J, Boyd M, Gilks C, Gartner C. Vaporised nicotine and tobacco harm reduction for addressing smoking among people living with HIV: A cross-sectional survey of Australian HIV health practitioners' attitudes. Drug Alcohol Depend 2017;177:67-70. doi:10.1016/j.drugalcdep.2017.03.023.

[35] Sigel K, Wisnivesky J, Shahrir S, Brown ST, Justice A, Kim J, et al. Findings in asymptomatic HIV-infected patients undergoing chest computed tomography testing: implications for lung cancer screening. AIDS 2014;28:1007-14. doi:10.1097/QAD.0000000000000189.

[36] DUBOIS C. Communiqué - Dépistage du cancer du poumon n.d. https://www.ifct.fr/index.php/fr/documentation/doc/item/2138-communique-depistagedu-cancer-du-poumon (accessed August 10, 2019).

[37] Lanoy E, Spano J-P, Bonnet F, Guiguet M, Boué F, Cadranel J, et al. The spectrum of malignancies in HIV-infected patients in 2006 in France : the ONCOVIH study. Int $\mathbf{J}$ Cancer 2011;129:467-75.

[38] Shiels MS, Althoff KN, Pfeiffer RM, Achenbach CJ, Abraham AG, Castilho J, et al. HIV Infection, Immunosuppression, and Age at Diagnosis of Non-AIDS-Defining Cancers. Clin Infect Dis 2017;64:468-75.

[39] Zeliadt SB, Heffner JL, Sayre G, Klein DE, Simons C, Williams J, et al. Attitudes and Perceptions About Smoking Cessation in the Context of Lung Cancer Screening. JAMA Intern Med 2015;175:1530-7.

[40] Iaccarino JM, Duran C, Slatore CG, Wiener RS, Kathuria H. Combining smoking cessation interventions with LDCT lung cancer screening: A systematic review. Prev Med 2019;121:24-32.

[41] Mercié P, Arsandaux J, Katlama C, Ferret S, Beuscart A, Spadone C, et al. Efficacy and safety of varenicline for smoking cessation in people living with HIV in France (ANRS 144 Inter-ACTIV): a randomised controlled phase 3 clinical trial. Lancet HIV 2018;5:e126-35.

[42] A S. Interventions for Tobacco Use Cessation in People Living With HIV and AIDS: Cochrane Nursing Care Field - Cochrane Review Summary. - PubMed - NCBI n.d. http://www.ncbi.nlm.nih.gov/pubmed/28034539 (accessed December 17, 2018).

[43] Jacquet J-M, Peyriere H, Makinson A, Peries M, Nagot N, Donnadieu-Rigole H, et al. Psychoactive substances, alcohol and tobacco consumption in HIV-infected outpatients. AIDS 2018;32:1165-71.

[44] Feuillet P, Lert F, Tron L, Aubriere C, Spire B, Dray-Spira R, et al. Prevalence of and factors associated with depression among people living with HIV in France. HIV Med 2017;18:383-94.

[45] Hartmann-Boyce J, McRobbie H, Bullen C, Begh R, Stead LF, Hajek P. Electronic cigarettes for smoking cessation. Cochrane Database Syst Rev 2016;9:CD010216.

[46] Hajek P, Phillips-Waller A, Przulj D, Pesola F, Myers Smith K, Bisal N, et al. A Randomized Trial of E-Cigarettes versus Nicotine-Replacement Therapy. N Engl J Med 2019;380:629-37. 
Tableau 1 : Etudes de dépistage

\begin{tabular}{|c|c|c|c|}
\hline & $\begin{array}{l}\text { Hulbert et al. [20] } \\
\text { Etats-Unis, 2014 }\end{array}$ & $\begin{array}{l}\text { Makinson et al } \\
\text { [21] France, 2016 }\end{array}$ & $\begin{array}{l}\text { Ronit et al.[22] } \\
\text { Danemark, 2017 }\end{array}$ \\
\hline $\mathrm{N}=$ & 224 & 442 & $\begin{array}{l}901 \text { dont } 113 \text { haut } \\
\text { risque** }\end{array}$ \\
\hline Age & $48(44-53)$ & $50(46-54)$ & $\begin{array}{l}50,4(43,5-59,0) \\
\text { Haut risque } 60,3(53,8- \\
65,2)\end{array}$ \\
\hline Hommes (\%) & $161(72 \%)$ & $370(84 \%)$ & $782(87 \%)$ \\
\hline $\begin{array}{l}\text { Tabac } \\
\text { F actifs } \\
\text { F sevrés } \\
\text { Non F }\end{array}$ & $\begin{array}{l}89 \% \\
11 \%\end{array}$ & $\begin{array}{l}92 \% \\
8 \% *\end{array}$ & $\begin{array}{l}27,9 \% \\
35,6 \% \\
34,4 \%\end{array}$ \\
\hline Paquets-années & 34 [31-36] & $30(25-40)$ & $18(6-32)$ \\
\hline Durée tabac (ans) & n.c. & $30(28-36)$ & n.c. \\
\hline TDM thoracique & $\begin{array}{l}\text { Base-line, } \quad \text { puis } \\
\text { 1x/an pendant } 4 \text { ans } \\
\mathrm{N}=678\end{array}$ & $\begin{array}{l}\text { Base-line } \\
\mathrm{N}=442\end{array}$ & $\begin{array}{l}\text { Base-line } \\
\mathrm{N}=902\end{array}$ \\
\hline $\begin{array}{ll}\text { Nombre } & \text { de } \\
\text { positifs à T0 } & \end{array}$ & $5 \%$ & $21 \%$ & $\begin{array}{l}3,1 \% \\
\text { Haut risque } 9,7 \%\end{array}$ \\
\hline $\begin{array}{l}\text { Nombre de CB à } \\
\text { T0 }\end{array}$ & $0 \%$ & $2,03 \%(8)$ & \\
\hline $\begin{array}{l}\text { Nombre de } \text { CB } \\
\text { détecté }\end{array}$ & $<0,01 \%(1 / 678)$ & $3,33 \%(9 / 442)$ & $\begin{array}{l}<1 \%(1 / 902) \\
\text { haut risque } 2,7 \% \\
(3 / 113)\end{array}$ \\
\hline $\begin{array}{l}\text { Nombre de } \mathrm{CB} \\
\text { non détecté }\end{array}$ & 0 & 1 & n.c. \\
\hline
\end{tabular}

$\mathrm{F}$ : fumeur ; n.c. : non connu ; *arrêt depuis 3 ans ;**âge >50 ans, Tabac >30 paquets-années 
Tableau 2: Données d'efficacité sur le sevrage tabagique d'essais randomisés issus de l'analyse Cochrane [30] chez les PVVIH comparant un bras interventionnel avec un soutien comportemental intensif et une pharmacothérapie à un bras contrôle avec une intervention comportementale brève et une pharmacothérapie.

\begin{tabular}{|l|l|l|l|l|}
\hline Objectif & Nbr d'études & Participants & Interventionnel vs standard & $\begin{array}{l}\text { Risque ratio } \\
\text { avec IC à 95\% }\end{array}$ \\
\hline Abstinence $>6$ mois & 6 & 1602 & $\begin{array}{l}8 \%(\mathrm{n}=61 / 760) \text { vs } 8 \% .(\mathrm{n} \\
=69 / 842)\end{array}$ & $1,00(0,72-1,39)$ \\
\hline Abstinence $<6$ mois & 11 & 1785 & $\begin{array}{l}13 \%(\mathrm{n}=118 / 937) \text { vs } 8 \% \\
(\mathrm{n}=67 / 848)\end{array}$ & $1,51(1,15-2)$ \\
\hline
\end{tabular}

IC : intervalle de confiance 
9

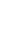

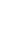

8

7

6

5

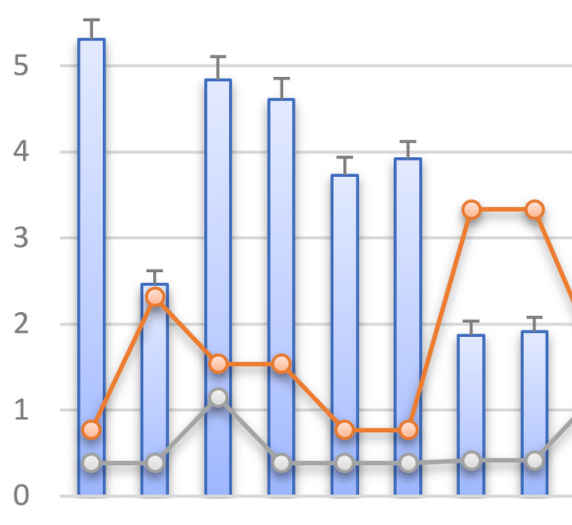

$\begin{array}{llllll}\mathrm{P} 1 & \mathrm{P} 2 & \mathrm{P} 3 & \mathrm{P} 4 & \mathrm{P} 5 & \mathrm{P} 6\end{array}$

\begin{abstract}
$\begin{array}{llll}\text { P7 } & \text { P8 } & \text { P9 } & \text { I1 }\end{array}$
\end{abstract}
$12 \quad 13$

$14 \quad 15$

$16 \quad 17$

I8 I9 AR1 AR2 AR3 AR4 AR5

$\square$ mean $\quad-$-floor effect $\quad-0$-ceiling effect 
Table 1. Patient characteristics at baseline $(n=26)$.

\begin{tabular}{|c|c|}
\hline Sex: male/female, n (\%) & $8(30.8) / 18(69.2)$ \\
\hline $\operatorname{Age}(y)^{*}$ & $48.9 \pm 13.7 / 50.0 \pm 13.3$ \\
\hline $\mathrm{BMI}\left(\mathrm{kg} / \mathrm{m}^{2}\right)$ & $24.7 \pm 3.1$ \\
\hline BDI-II & $5.3 \pm 5.0$ \\
\hline FSS ( /7) & $4.9 \pm 1.3$ \\
\hline CMTNS ( /36) & $14.6 \pm 5.2$ \\
\hline CMTNS SS ( /12) & $3.3 \pm 1.7$ \\
\hline CMTNS ES ( /28) & $10.0 \pm 4.1$ \\
\hline INCAT Sensory Sum score ( /20) & $6.4 \pm 3.8$ \\
\hline INCAT sensory score on lower limb ( /12) & $3.2 \pm 1.5$ \\
\hline Orthopaedic shoes / AFOs / none, n (\%) & $22(84.7) / 3(11.5) / 1(3.8)$ \\
\hline Pes cavus type 1 / type 2 / type 3 , n (\%) & $24(46.2) / 20(38.5) / 8(15.3)$ \\
\hline Flexible / Rigid Hammertoes / none, n (\%) & $153(58.8) / 93(35.8) / 14(5.4)$ \\
\hline History of foot surgery, $\mathrm{n}(\%)$ & $5(19.2)$ \\
\hline FFI & $83.1 \pm 36.4$ \\
\hline Pain & $33.6 \pm 15.3$ \\
\hline Disability & $41.6 \pm 17.8$ \\
\hline Activity restriction & $10 \pm 8.3$ \\
\hline SF-36 physical composite score & $36.3 \pm 9.6$ \\
\hline SF-36 mental composite score & $47.8 \pm 11.6$ \\
\hline Speed $(\mathrm{cm} / \mathrm{s})$ & $91.6 \pm 23$ \\
\hline Cadence (steps/min) & $103.9 \pm 13.7$ \\
\hline Stride length $(\mathrm{cm})$ & $104.7 \pm 17.1$ \\
\hline \multicolumn{2}{|l|}{ Peak torque $(\mathrm{Nm} / \mathrm{Kg})$} \\
\hline \multicolumn{2}{|l|}{ Knee flexors } \\
\hline $120^{\circ} / \mathrm{s}(\mathrm{conc})$ & $34.6 \pm 19.6$ \\
\hline $60 \% / \mathrm{s}(\mathrm{conc})$ & $47.5 \pm 23.9$ \\
\hline $30 \% \mathrm{~s}(\mathrm{ecc})$ & $94 \pm 41.7$ \\
\hline \multicolumn{2}{|l|}{ Knee extensors } \\
\hline $120 \%$ s (conc) & $45.2 \pm 23.8$ \\
\hline $60 \%$ s (conc) & $67 \pm 31.5$ \\
\hline $30 \% / \mathrm{s}(\mathrm{ecc})$ & $135.1 \pm 47.6$ \\
\hline
\end{tabular}

Data are mean \pm SD unless indicated.

* mean \pm SD / median \pm SD.

BMI, body mass index; BDI-II, second version of the beck depression inventory; FSS, fatigue severity scale; CMTNS, Charcot-Marie-Tooth neuropathy score; CMTNS ES, exam composite score of the CMTNS; CMTNS SS, symptom composite score of the CMTNS; INCAT: Inflammatory Neuropathy Cause and Treatment; AFOs, ankle foot orthotics; FFI, Foot Function Index; SF-36, Medical Outcomes Study Short Form 36 health-related quality of life; conc, concentric mode; ecc, eccentric mode. 